\section{MRS Bulletin Volume Organizers guide technical theme topics for 2020}

\author{
www.mrs.org/bulletin
}

$\mathrm{T}^{\mathrm{h}, \mathrm{w}}$ he MRS Bulletin 2020 Volume Organizers, appointed by MRS President Michael R. Fitzsimmons, will guide the development of theme topics for the 2020 volume year. They are Hongyou Fan (Sandia National Laboratories and The University of New Mexico, USA), Oleg Gang (Columbia University and Brookhaven National Laboratory, USA), Seokwoo Jeon (Korea Advanced Institute of Science and Technology, Republic of Korea), and Tae-Woo Lee (Seoul National University, Republic of Korea).

Hongyou Fan is a distinguished member of the Technical Staff at Sandia National Laboratories and is a national laboratory professor in the Department of

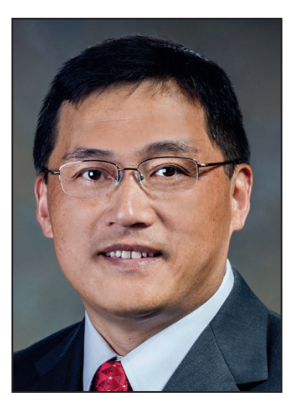

Chemical and Biological Engineering at The University of New Mexico. He earned his $\mathrm{PhD}$ degree from The University of New Mexico in chemical engineering. He was a postdoctoral fellow at Sandia National Laboratories, Albuquerque, N.M., before working there full-time. His current research focuses on the development of active nanomaterials for nanoelectronic and nanophotonic applications. Fan is a Fellow of the American Physical Society and MRS, and he has received the MRS Kavli Distinguished Lectureship Award in Nanoscience, an R\&D 100 Award for the development of technically significant products, the Federal Laboratory Consortium for Technology Transfer-Outstanding Technology Development Award, The University of New Mexico Outstanding Faculty Mentor Award, and the Asian American Engineer of the Year Award.

Oleg Gang is a professor of chemical engineering and of applied physics and materials science at Columbia University, and is a leader of the Soft and Bio Nanomaterials Group at Brookhaven National
Laboratory (BNL). His research explores the behavior of soft matter at the nanoscale, and he develops novel strategies

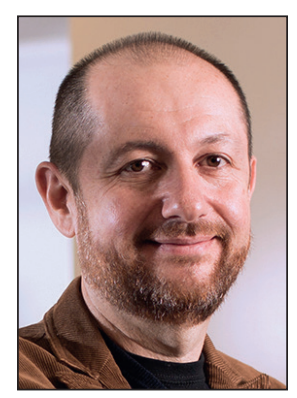

for programmable assembly of targeted nanomaterials with designed spatial organizations. Gang earned a $\mathrm{PhD}$ degree from Bar-Ilan University, was a postdoctoral Rothschild Fellow at Harvard University, and was a Distinguished Goldhaber Fellow at BNL, where he became a leader of the Soft and Bio Nanomaterials Group at the Center for Functional Nanomaterials. In 2016, Gang joined the faculty at Columbia University. His group develops new strategies for creating materials by design using nano-assembly approaches, as well as employs advanced in situ probes for understanding structure formation. Gang has received numerous awards and recognitions, including the Gordon Battelle Prize for Scientific Discovery and the US Department of Energy Outstanding Mentor Award, and he is a Fellow of the American Physical Society.

Seokwoo Jeon is a chair professor of materials science and engineering at the Korea Advanced Institute of Science and Technology (KAIST). He is also the

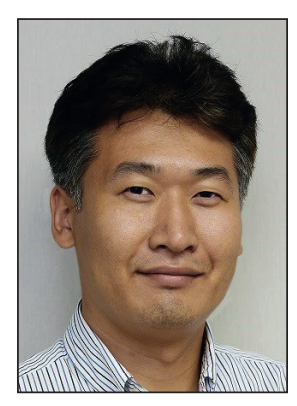
director of the Advanced MEMS GC Center for Drug Detection Project, granted by the Korea Police Department, and a founding member of the Young Korean Academy of Science and Technology. He received a $\mathrm{PhD}$ degree in materials science and engineering in 2006 from the University of Illinois at Urbana-Champaign. Jeon's main research goals are exploring unique electronic, mechanical, and optical properties of novel nanomaterials and employing those materials in real-world applications. Currently, his group specializes in the synthesis of low-dimensional materials, including graphene, graphene quantum dots, and $\mathrm{WS}_{2}$, and fabrication of metallic or ceramic 3D nanostructures, all of which demonstrate potential in various applications. Jeon has made many contributions in the field of nanotechnology. He is a board member of numerous academic societies and session organizer or member of an organizing committee of many international conferences, such as the International Committee on Composite Materials, the Asian-Australian Conference on Composite Materials, and MRS. He won the Presidential Young Scientist Award from the Republic of Korea in 2015.

Tae-Woo Lee is an associate professor in the Department of Materials Science and Engineering at Seoul National University. He received his $\mathrm{PhD}$ degree in chem-

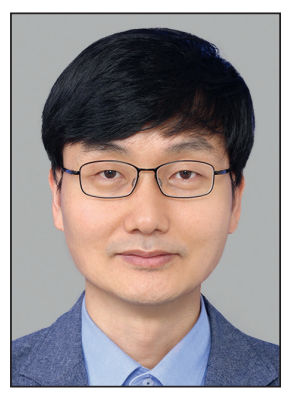

ical engineering from the Korea Advanced Institute of Science and Technology in February 2002. He joined Bell Laboratories as a postdoctoral researcher and later worked at the Samsung Advanced Institute of Technology, Samsung Electronics, as a member of the research staff. From August 2008 to August 2016, Lee was an assistant and then an associate professor in the Department of Materials Science and Engineering at Pohang University of Science and Technology, Republic of Korea. He received the Korea Young Scientist Award from the president of Korea in 2008, and The Scientist of the Month Award from the Ministry of Science, ICT and Future Planning in 2013. He is an author or co-author of more than 180 papers, as well as an inventor and co-inventor of 357 patents. Lee's research focuses on organic, organicinorganic hybrid perovskites, and carbon materials and their applications to flexible electronics, printed electronics, displays, solid-state lighting, solar-energy-conversion devices, and neuromorphic devices. 